\title{
BLICKDIAGNOSE
}

Non-Hodgkin-Lymphom

\section{Woher kommt die Dyspnoe?}

Dem 62-jährigen, männlichen Patienten wurde im April 2009 die Diagnose eines großzelligen Non-Hodgkin-Lymphoms mit spinalem Befall gestellt. Es erfolgten die Tumorexstirpation mittels Hemilaminektomie, eine postoperative Bestrahlung mit einer Gesamtdosis von 40 $G y$ und die Einleitung einer Chemotherapie nach dem R-CHOP-21-Protokoll. Aufgrund einer Paraparese beider Beine wurde eine neurologische Rehabilitation initiiert. Während dieser Rehabilitation verschlechterte sich Anfang August 2009 der Allgemeinzustand des Patienten. Es trat eine Dyspnoesymptomatik ein.

- Im weiteren Verlauf gelang der klinische und paraklinische Nachweis einer beidseitigen Pneumonie. In der Computertomografie zeigte sich neben bekannten Bullae der Oberlappen ein fibrotischer Umbau der Lunge nach Bestrahlung. Ferner stellte sich das Portsystem des Patienten entzündet dar. In der bronchoalveolären Lavage gelang der mikrobielle Nachweis von Candida glabrata, Corynebacteriae spp. und Proteus mirabilis. In der Blutkultur ließ sich Staphylococcus epidermidis nachweisen. Es erfolgte eine resistenzgerechte antibiotische und antimykotische Therapie sowie die Entfernung des Portsystems.

Im weiteren Verlauf trübte der Patient bei respiratorischer Globalinsuffizienz und septischem Krankheits-

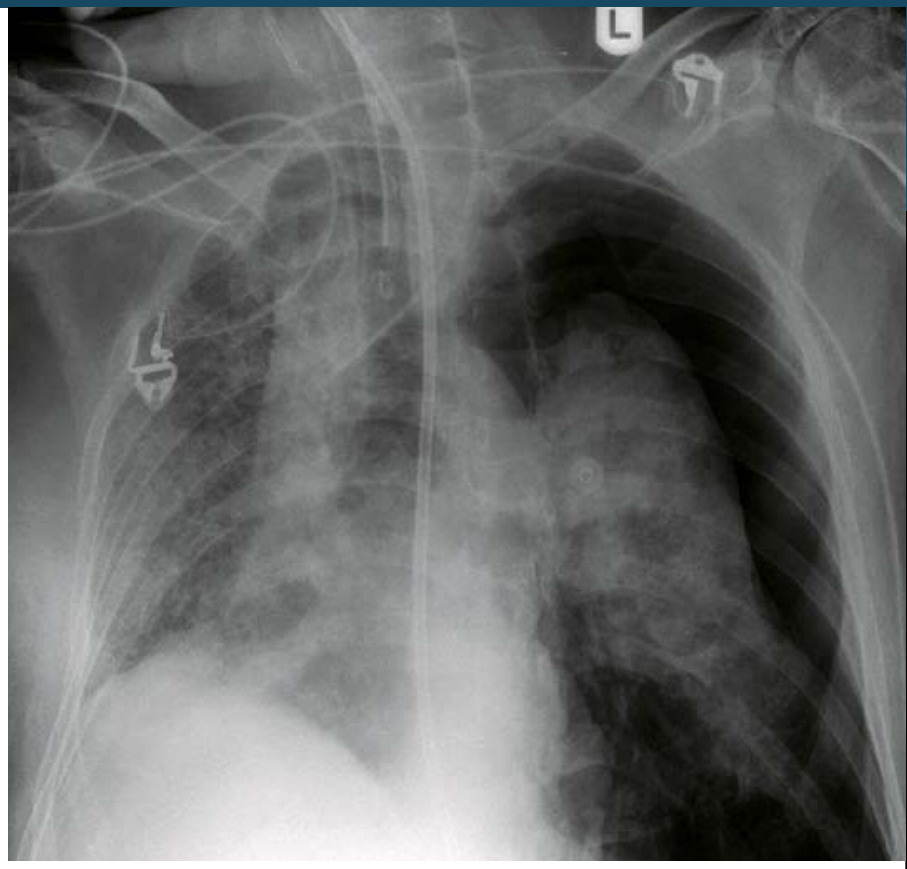

verlauf soporös ein und musste am 5.8.2009 intubiert und mit zunächst normalem Druck (CPAP $5 \mathrm{mBar}$ ) beatmet werden. Aufgrund weiterer respiratorischer Verschlechterung am 7.8.2009 mit im Verlauf ansteigendem Beatmungsdruck (CPAP $18 \mathrm{mBar}$ ) und niedriger Compliance wurde erneut eine Röntgen-Thorax-Aufnahme angefertigt, in der sich das Bild eines linksseitigen Spannungspneumothorax zeigte. Die Anlage einer Thoraxsaugdrainage und Besaugung über 15 Tage führte nicht zur Sanierung des Pneumothorax. In der am 21.8.2009 durchgeführten Lungenteilresektion $\mathrm{S} 1$ links ließ sich eine Lungenfistel darstellen und wurde operativ verschlossen.

Keywords: tension pneumothorax, pulmonary fistula

- Dr. med. Joachim Känel, Dr. med. Harald Schmalenberg, Dr. med. Rüdiger Pfeifer, Prof. Dr. med. Hans-R. Figulla, Dr. med. Christian Jung, Klinik für Innere Medizin I der Universitätsklinik Jena, Erlanger Allee 101, D-07747 Jena

\section{Ihr besonderer Fall?}

\section{Stellen Sie uns Ihren Fall vor}

Sicher sehen auch Sie ab und an einen besonders eindrucksvollen Befund in Ihrer Praxis. Fotografieren Sie ihn, schreiben Sie uns unter dem Stichwort Blickdiagnose, bei Veröffentlichung erhalten Sie 100 Euro.

MMW-Fortschritte der Medizin

E-Mail: reinhold.manhart@springer.com

Fax: 089/203043-31424 